consistently associated with an excess mortality. Previous analyses in MWS found smoking 15 cigarettes per day was associated with about a $180 \%$ excess risk of mortality compared to never smokers $(R R=2.77,2.72-2.82)$.

Conclusion While social isolation was associated with about a $30-40 \%$ excess risk of all-cause mortality, the excess risk associated with smoking 15 cigarettes per day was 4-6 fold greater, at around $180 \%$.

\section{P85 QUANTIFYING SOCIAL INEQUALITIES IN SERIOUS INFECTIONS IN ENGLAND: A LONGITUDINAL ECOLOGICAL ANALYSIS OF HOSPITAL EPISODE STATISTICS DATA}

1,2,3,4TE Wingfield, ${ }^{5} \mathrm{~T}$ Rose, ${ }^{5} \mathrm{~B}$ Barr, ${ }^{5} \mathrm{D}$ Taylor-Robinson*. ${ }^{1}$ Clinical Sciences and
International Public Health, Liverpool School of Tropical Medicine, Liverpool, UK; ${ }^{2}$ Institute
of Infection and Global Health, University of Liverpool, Liverpool, UK; ${ }^{3}$ Social Medicine,
Infectious Diseases, and Migration, Karolinska Institutet, Stockholm, Sweden; ${ }^{4}$ Tropical and
Infectious Diseases Unit, Royal Liverpool and Broadgreen University Hospitals NHS Trust,
Liverpool, UK; ${ }^{5}$ Institute of Population Health Sciences, University of Liverpool, Liverpool, UK

\subsection{6/jech-2019-SSMabstracts.235}

Background In England, infections cause $7 \%$ of deaths, cost 30 billion pounds annually, and are more common in disadvantaged populations. However, there has been no single, unifying analysis of the overall social patterning of the health burden of infections in England and how this is changing over time. This study quantified social inequalities in patients admitted to secondary healthcare facilities for infections in England between 2005 and 2016.

Methods An ecological analysis was performed using neighbourhood-level Hospital Episode Statistics (HES) data concerning emergency hospitalisations across England for the study period from 2005 to 2016. Linear mixed effects models were used to assess age-adjusted trends in emergency admission rates for serious infections. Absolute inequalities in emergency admission rates for serious infections between the most and least income deprived neighbourhoods were quantified and compared to trends in non-infectious disease related admissions.

Results Between 2005 and 2016, age-adjusted emergency admission rates for serious infections increased 52\% (1894 and 2870 per 100,000 population for 2005 and 2016, respectively) across England. In 2005, the absolute inequalities gap comparing rates of serious infections in the highest versus lowest deprivation quintile was 1197 per 100,000 (95\%CI 1176 to 1217 ) and this increased over time. The annual increase in absolute inequality in the most deprived areas compared to the least was 51 emergency admissions per 100,000 (95\%CI 49-52). In 2005, the absolute inequalities gap comparing rates of non-infectious disease related admissions in the highest versus lowest deprivation quintile was 5950 per 100,000 (95\%CI 5880 to 6020$)$. The annual increase in absolute inequality of non-infectious disease related admissions in the most deprived areas compared to the least was 27 emergency admissions per 100,000 (95\%CI 22-32). Subsequent analyses will explore trends in different age groups, alternative measures of inequalities, and sub-groups of infectious outcomes.

Discussion Emergency admission rates for serious infections and non-infections have increased in England. In addition, absolute inequalities have widened, especially for emergency admissions due to serious infections. These findings support the need for stronger public health and prevention efforts to address inequalities in the social determinants of ill health, with a specific focus on infectious diseases.

\section{P86 INTERPRETING THE CHANGING ASSOCIATION BETWEEN CAESAREAN BIRTH AND NEONATAL DEATH: A CASE STUDY FROM ETHIOPIA}

${ }^{1} \mathrm{E}$ Yisma*, ${ }^{2} \mathrm{~B} \mathrm{Mol},{ }^{1} \mathrm{~J}$ Lynch, 'L Smithers. 'School of Public Health, The University of Adelaide, Adelaide, Australia; '2Department of Obstetrics and Gynaecology, Monash University, Melbourne, Australia

\subsection{6/jech-2019-SSMabstracts.236}

Background Both individual- and aggregate-level studies have yielded inconsistent results about the association between caesarean birth and neonatal mortality. We provide an interpretation of the changing association over time between caesarean birth and neonatal death using Demographic and Health Survey (DHS) data within the context of Ethiopia.

Methods We used data from Ethiopian DHS in 2000, 2005, 2011, and 2016. We analysed the association between caesarean birth and neonatal death using log-Poisson regression models for each survey adjusted for potential confounders. We then applied the 'Three Delays Model' to provide an interpretation of the changing association between caesarean birth and neonatal death in Ethiopia.

Results The adjusted prevalence ratios (aPR) for neonatal death among neonates born via caesarean section versus vaginal birth increased over time, from 0.95 (95\% CI, 0.29, 3.19) in 2000 to $2 \cdot 81(95 \% \mathrm{CI}, 1 \cdot 11,7 \cdot 13)$ in 2016 . The association between caesarean birth and neonatal death was stronger among rural women (aPR (95\% CI) $3.43(1.22,9.67)$ ) and among women from the lowest quintile of household wealth (aPR (95\% CI) $7 \cdot 01(0 \cdot 92,53 \cdot 36))$ in 2016. However, the aggregate-level analysis revealed that an increase in caesarean section rate is correlated with a decrease in the proportion of neonatal deaths.

Conclusion The naïve interpretation of the changing association between caesarean birth and neonatal death from 2000 to 2016 is that caesarean section is increasingly associated with neonatal death. However, the changing association reflects improvements in health service coverage and a shift in the characteristics of Ethiopian women undergoing caesarean section after complicated labour or severe foetal compromise. 